\title{
The Research of Anti Collision Algorithm in the Clothing Identification Based on RFID
}

\author{
Xiao Luo ${ }^{1, a}$, Fei Guo ${ }^{2, b}$, Xiaojie Liu ${ }^{3, c}$, Ning He ${ }^{4, d}$, Yanping Wang ${ }^{5, e}$ \\ 1,2,3,4,5 Department of Information Engineering, Beijing Institute of Fashion Technology, Beijing, \\ 100029, China \\ aemail: Ixiaorow@yeah.net
}

Keywords: RFID; Clothing Identification; Anti Collision Algorithm

\begin{abstract}
Radio frequency identification (RFID) is a kind of automatic identification technology; it can easily finish automatic data acquisition and recognition by the way of noncontact reading. In the clothing industry, the clothing logo includes much more information and has many problems such as the low speed of artificial input, bad efficiency, and poor interaction interface. In order to adapt to the modern clothing enterprise mass production and the demands of the sale, this paper proposes the new clothing identification system which is based on RFID. The basic information of the clothing tags is stored in the RFID and realized the clothing information management and display. Because RFID technology can identify more than one targets at one time, the anti collision algorithm becomes the key technology and the prerequisite. Therefore, this paper puts forward the improved algorithm based on the research of traditional anti collision algorithm. By the simulation, the performance of the new algorithm is better than the traditional ones.
\end{abstract}

\section{Introduction}

RFID (Radio Frequency Identification) has been applied in various fields in the late 20th century[1]. It is a kind of non-contact automatic Identification technology. Using wireless Radio Frequency signal through space coupling (electromagnetic induction and electromagnetic propagation mode), it can realize non-contact two-way data transmission, and get the relevant data, so as to achieve the aim of automatic Identification target object. Comparing with the bar code, IC card, magnetic stripe, fingerprints and optical character, RFID technology has huge advantages in the field of long identification distance[2], high accuracy, durable, large storage capacity, environmental requirements, non-contact and multiple labels can identify at the same time.

In the field of clothing production, the basic content of a costume generally always includes name, manufacturer, model size, fabric content, washing method, matters needing attention, national standard and the quality grade. The information is huge. Aiming at defects of clothing identification methods, this paper puts forward a kind of clothing identification method based on RFID technology, and the efficiency of recognition is also researched. The scheme is realized by the internal transponder embedded clothing as data carrier[3]. It can identify and manage the appropriate clothing materials. The clothes with labels can be recognized and monitored in the entire clothing production and sales process. The clothing identification technology which is discussed in this paper is to improve the current defects and deficiencies.

At present, RFID technology research is mainly focus on frequency selection, antenna design, collision technology, security and privacy protection.

(1)The choice of working frequency: It should not only meet the needs of various applications, but also need to consider the use of radio spectrum and the rules of the transmitted power in different countries. The current RFID working frequency spans multiple frequencies[4]. Different frequency bands have their advantages and disadvantages, it impacts on the performance of the label and size, also affects the price of tags and readers.

(2)RFID antenna research: At present, the studies of RFID antenna are mainly concentrated in the antenna structure and environmental factors. Antenna structure can decide the direction of the antenna diagram, polarization direction, impedance characteristics, standing wave ratio, antenna 
gain and frequency characteristics. And the characteristic of the antenna is affected by the shape and physical properties of an identified object, and it is also influenced by antenna objects and surroundings.

(3)Collision technology research: Because the multiple RFID tags are at the same frequency, information transmission process will produce conflict when they are in the work area of the same reader[5]. Work area overlap between multiple readers at the same time will also cause conflicts.

(4)Security and privacy issues: They are focused on privacy protection for individual users and the enterprise business.

For the clothing identification system, when $\mathrm{N}$ tags are in a reader function scope, there are two kinds of communication mode.

The first is from reader to tags and from the tag to the reader. This kind of data transmission is also known as "radio"; the second is within the scope of the reader, there are multiple tags at the same time to transmit data to the reader, and it is called multiple accesses. In the case of multiple accesses, with different signal aliasing and interference, the reader cannot identify any labels. This paper will also focus on this kind of conflict.

\section{System Solutions}

Firstly, as the most basic identity information, the serial number will be written to the clothing RFID carrier. Then, in the clothing production process this part will be embedded in clothing, such as a collar, cuff or inside seam and etc. Through the software system its corresponding identity information can be store in the database system based on Internet. In the clothing circulation field, combing with the reader and network database resource, the identity information can be efficiently managed.

(l)The transponder: Transponder (electronic tags) is the real data carrier in RFID system. In general, transponder has no power supply (or battery); only in the range of reader's response it is active. Transponder power is needed to work as a clock and data through the coupling unit of transmission to the transponder. Transponder is usually composed with the microchip and the coil in a large area of the antenna. As clothing identification transponder can have various types of packaging, but its internal structure is basic same. For most of the clothes, the labels are on the buttons.

(2)RFID Read/write device: The basic task is to start and communicate with the transponder. The device has the read/write function. Reader is divided into fixed and handheld one. The reader reads the data through the antenna sensor tags. The read distances of fixed reader can be up to 8 meters, the handheld reader is about $1 \mathrm{~m}$.

(3)RFID Middleware software: It is responsible for hardware and software communication system.

(4)Software System: In the clothing field, the main management of the system is clothing identification and circulation information, including the purchase process, sales and inventory. Considering the safety of the system, it also should include user management functions.

\section{Tag anti-collision algorithm}

(1)The commonly used algorithm

In wireless communication system, the methods to solve the conflict are usually divided into the following categories:

A. FDMA: it supplies different carrier frequency transmission channel for user at the same time. Normally, the frequency of RFID system from the reader to the tag is fixed. From the electronic tag to the reader, tags can use different and independent subcarrier frequency to transmit data. The defect is expensive, because each receiving channel has separate receiver for use, so this conflict prevention methods are restricted to a few special applications.

B. SDMA: It can realize the multiple target recognition within the scope of the scattered space. Generally by two methods, one is on the reader, with the phased array antenna and the antenna 
directivity diagram on an electronic tag, different labels can be identified by the difference angle position within the scope of the reader; another way is to divide the space of reader and antenna range. When the label is into the different reader range, it can be differentiated from the space. Its main drawback is the complex antenna system and the high cost of implementation, and not easy to achieve, so the special occasions generally adopts SDMA.

C. TDMA: according to the time allocate to multiple users. TDMA is firstly used in the digital mobile system. TDMA can be divided into electronic label drive method and the reader driver method. This article focuses on the tag anti-collision identification method.

(2)Binary algorithm

Binary search algorithm is the anti-collision algorithms in RFID system. Compared with the random search algorithm, it has good reliability, channel using fully, especially more tag number. The algorithm is the kind of essentially successive comparison method. It selects different serial number, exchanges data gradually between the reader and electronic tag through the time-division multiplex method. As a result, the average comparison number can be represented as:

$$
L=\log _{2} N+1
$$

(3)The basic principle of improved algorithm

The algorithm is divided into three conditions: the first is when reader sends a Request command and returns no collision; the second is the collision has happened when the serial number is the highest level of 0 . The third case is the collision serial number of 1 . As a result, the main process of the improved binary collision algorithm is as follows:

A. Reader sends the Request, all tags in the covering area response and send the ID value synchronous back to the reader.

B. Reader detects the received data. If it is zero, the tag number is 0 , it can return to the first step, otherwise go to $(\mathrm{C})$.

C. Using the received data, if there has no collision, there is only a tag within the range. Send "Select" and "Read-Write" operations to the tag and finally send "Unselect" command. If there has collision, record the ID value firstly, and then go to the (D).

D. Reader tests ID value, the bits which have no collision set zero, otherwise set 1 , component command Request (ID, 0) in the first parameter, and the echo command to all tags within the scope of cover. The new serial number has been made. If the first part of new serial number is 0 , then return all the remaining bits to the reader. Otherwise, no response. The reader judges whether there has a collision, if not, then send the "Select" and the "Read-Write" command. Finally, send "Unselect" command, and then transferred to (E). If collision has occurred, it began to collision detection and set 0 to the highest bit. Using the highest bit of the binary number as the Request parameter (ID), continue to send. After dealing with a tag, the algorithm adopts the back tactics; identifies another branch of this node, repeated iteration operation, until all tags are identified.

E. Reader sends the Request (1) command. Label return to the rest of the top bits, reader detects data, if there has no collision, then send "Select" and "Read-Write" command and finally send "Unselect" command tags, then go to (F).

F. all tags are identified within the scope of reader, the algorithm is over.

(4)The comparison and performance analysis of improved algorithm

A. BS algorithm: Assuming the $\mathrm{k}$ bits UID, the length of the label sends UID for $\mathrm{k}$, we can calculate the tag and reader transfer the sum of all the bit length $\mathrm{L}_{\mathrm{BS}}$, and the total transmission time delay $\mathrm{T}_{\mathrm{BS}}$ for $\mathrm{n}$ labels as follows:

$$
L_{B s}=n \cdot\left[(2 k+21) \cdot \operatorname{Integ}\left(\log _{2} n\right)+(2 k+23)\right]
$$

$$
T_{B S}=\frac{L_{B S}}{v}=\frac{n \cdot\left[(2 k+21) \cdot \operatorname{Integ}\left(\log _{2} n\right)+(2 k+23)\right]}{v}
$$

B. DBS algorithm: LDBS and TDBS is as follows:

$$
\begin{aligned}
L_{D B S} & =(2 k+21) n+(k+21)\left[n \cdot \operatorname{Integ}\left(\log _{2} n\right)-n\right]+(k+23) n \\
& =(3 k+44) n+(k+21)\left[n \cdot \operatorname{Integ}\left(\log _{2} n\right)-n\right] \quad T_{B S}=\frac{L_{D B S}}{\nu}=\frac{(3 k+44) n+(k+21)\left[n \cdot \text { Integ }\left(\log _{2} n\right)-n\right]}{\nu}
\end{aligned}
$$

C. The improved binary algorithm: 


$$
\begin{aligned}
L_{N E W} & =(2 k+21)+(k+x+21)+(x+23) n+(x+21)(n-3) \\
& =3 k-2 x-21+(2 x+44) n
\end{aligned}
$$

$$
\begin{array}{ll}
T_{N E W}=\frac{L_{N E W}}{\nu}=\frac{3 k-2 x-21+(2 x+44) n}{\nu} & (n \geqslant 2) \\
T_{N E W}=\frac{L_{N E W}}{v}=\frac{2 k+23}{\nu} & (n=1)
\end{array}
$$

Through figure 1 we can see the time delay in improved algorithm of is much less than the original binary algorithm and dynamic binary algorithm.
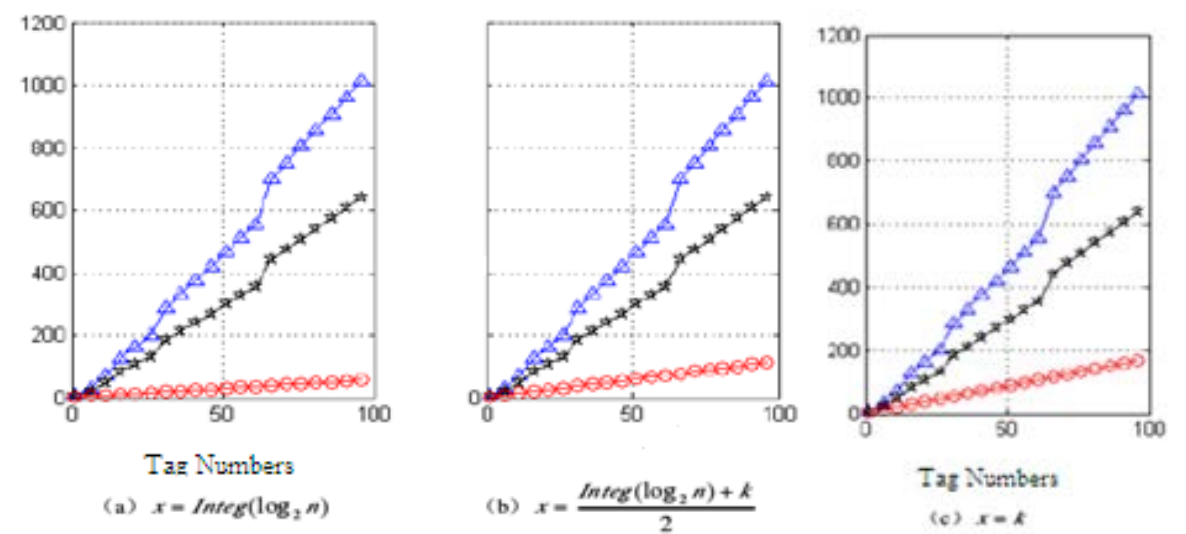

Fig.1 The improved algorithm transmission delay chart

\section{Conclusion}

In this paper, we put forward to apply RFID technology to the traditional garment field. And based on the collision problem, we realize the corresponding improved binary algorithm. This paper analyzes the ID value between the tag and reader. It is concluded that the differences of tags within range are due to the value of the corresponding different collision. With the improved algorithm, we set a new ID for the collision, send back binary number to the reader before the collision. The data is as a new recognition object to carry on the back. Through the experimental results show that the improved algorithm is more suitable for the much tag number, longer ID length. Through the $L_{B S}$ and $\mathrm{T}_{\mathrm{BS}}$ simulation, the improved algorithm which is proposed in this paper has great superiority comparing with the original binary search algorithm and dynamic binary search algorithm.

\section{Acknowledgement}

In this paper, the research was sponsored by the General Program of Science and Technology Development Project of Beijing Municipal Commission of Education (Project No. KM201410012001) and Teaching Plan and Youth Innovation Fund Project of science of Beijing Institute Of Fashion Technology (Project No.JG-1414 and 2014AL-29)( PTTBIFT_YC_009).

\section{References}

[1] Finkenzeller K., "RFID Handbook," John Wiley and Sons, 1999.

[2] Juels A., "RFID security and privacy: a research survey," IEEE transaction on Selected Areas in Communications, Vol. 24, Issue 2, pp. 381-394, Feb. 2006.

[3] P.Bahl and V.N.Padmanabhan, "RADAR: An in-building RF-based user location and tracking system," in Proc. IEEE INFOCOM 2000, Mar., vol.2, pp.775-784.

[4]Yiyang Zhao, Yunhao Liu, Lionel M. Ni, "VIRE: Active RFID-based Localization Using Virtual Reference Elimination," icpp, pp.56, 2007 International Conference on Parallel Processing (ICPP 2007), 2007.

[5] S.Polito, D.Biondo, A.Iera, M.Mattei and A.Molinaro, "Performance evaluation of active RFID location systems based on RF power measures," Personal, Indoor and Mobile Radio Communications, 2007., pp.1-5, Sept.2007. 